\title{
On the number of paths of lengths 3 and 4 in a graph
}

\author{
Nazanin Movarraei*, M. M. Shikare \\ Department of Mathematics, University of Pune, Pune 411007 (India) \\ *Corresponding author E-mail: nazanin.movarraei@gmail.com
}

Copyright (C)2014 Nazanin Movarraei, M. M. Shikare. This is an open access article distributed under the Creative Commons Attribution License, which permits unrestricted use, distribution, and reproduction in any medium, provided the original work is properly cited.

\begin{abstract}
In this paper, we obtain explicit formulae for the total number of paths of lengths 3 and 4 in a simple graph G. We also determine some formulae for the number of paths of lengths 3 and 4 each of which starts from an specific vertex $v_{i}$ and for the number of $v_{i}-v_{j}$ paths of lengths 3 and 4 in a simple graph $\mathrm{G}$, in terms of the adjacency matrix and with the helps of combinatorics.
\end{abstract}

Keywords: Adjacency Matrix, Cycle, Graph Theory, Path, Subgraph, Walk .

\section{Introduction}

In a simple graph $\mathrm{G}$, a walk is a sequence of vertices and edges of the form $v_{0}, e_{1}, v_{1}, \ldots, e_{k}, v_{k}$ such that the edge $e_{i}$ has ends $v_{i-1}$ and $v_{i}$. A walk is called closed if $v_{0}=v_{k}$. If the vertices of a walk are distinct then that walk is called a path and a cycle is a non-trivial closed path.

It is known that if a graph $\mathrm{G}$ has adjacency matrix $\mathrm{A}=\left[a_{i j}\right]$, then for $\mathrm{k}=0,1, \ldots$, the ij-entry of $\mathrm{A}^{k}$ is the number of $v_{i}-v_{j}$ walks of length $\mathrm{k}$ in $\mathrm{G}$. It is also known that $\operatorname{tr}\left(\mathrm{A}^{n}\right)$ is the sum of the diagonal entries of $\mathrm{A}^{n}$ and $d_{i}$ is the degree of the vertex $v_{i}$.

In 1971, Frank Harary and Bennet Manvel [1], gave a formula for the number of triangles in simple graphs as given by the following theorem:

Theorem 1.1 If $G$ is a simple graph with adjacency matrix $A$, then the number of 3 -cycles in $G$ is $\frac{1}{6}$ tr $\left(A^{3}\right)$.

(It is known that $\operatorname{tr}\left(A^{3}\right)=\sum_{i=1}^{n} a_{i i}^{(3)}=\sum_{i \neq j} a_{i j}^{(2)} a_{i j}$ ).

They also gave formulae for the number of cycles of lengths 4 and 5 in simple graphs. Their proofs are based on the following fact:

The number of $n$-cycles $(n=3,4,5)$ in a graph $\mathrm{G}$ is equal to $\frac{1}{2 n}\left(\operatorname{tr}\left(\mathrm{A}^{n}\right)-\mathrm{x}\right)$ where $\mathrm{x}$ is the number of closed walks of length $\mathrm{n}$, which are not n-cycles.

In 1986, Tomescu [2], gave some formulae for the number of paths of length s having $\mathrm{k}$ edges in common with a fixed s-path of a complete graph. In 1994, Bax [3], gave an algorithm to count number of all paths and $v_{i}-v_{j}$ paths in a graph. His algorithm was about counting number of all paths in a graph and it can not count number of paths of an specific size. 
In 1996, Eric Bax and Joel Franklin [5], gave an algorithm to count paths and cycles of a given length in a directed graph. In [4], [6], [7], [8], [10], [11] and [13], we have also some bounds to estimate the total time complexity for finding or counting paths and cycles in a graph.

In the previous works there is no formula to count the exact number of paths of an specific size in a graph.

In this paper we give some formulae to count the exact number of paths of lengths 3 and 4 in a simple graph $G$, in terms of the adjacency matrix of $\mathrm{G}$ and with the helps of combinatorics.

We state the following propositions which are useful to prove our theorems:

Proposition 1.2 In a simple graph $G$ with $n$ vertices and the adjacency matrix $A=\left[a_{i j}\right]$, the number of paths of length $n$ is $\sum_{i \neq j} a_{i j}^{(n)}-x$, where $x$ is the number of non-closed walks of length $n$ in $G$, which are not paths.

Proposition 1.3 In a simple graph $G$ with $n$ vertices and the adjacency matrix $A=\left[a_{i j}\right]$, the number of paths of length $n$, each of which begins with an specific vertex $v_{i}$ is $\sum_{j=1, i \neq j}^{n} a_{i j}^{(n)}-x$, where $x$ is the number of non-closed walks of length $n$ in $G$, starting from the vertex $v_{i}$, which are not paths.

Proposition 1.4 In a simple graph $G$ with $n$ vertices and the adjacency matrix $A=\left[a_{i j}\right]$, the number of $v_{i}-v_{j}$ $(i \neq j)$ paths of length $n$ is $a_{i j}^{(n)}-x$, where $x$ is the number of non-closed $v_{i}-v_{j}$ walks of length $n$ in $G$, which are not paths.

\section{Number of paths of length 2}

Let $\mathrm{G}$ be a simple graph with $\mathrm{n}$ vertices and the adjacency matrix $\mathrm{A}=\left[a_{i j}\right]$, then the following results are known by [1] :

1. The number of paths of length 2 in $\mathrm{G}$ is $\sum_{i \neq j} a_{i j}^{(2)}$, which is also equal to $\sum_{i \neq j} a_{i j}\left(d_{j}-1\right)$.

2. The number of paths of length 2 in $\mathrm{G}$, each of which starts from the vertex $v_{i}$ is $\sum_{j=1, i \neq j}^{n} a_{i j}^{(2)}$, which is also equal to $\sum_{j=1, i \neq j}^{n} a_{i j}\left(d_{j}-1\right)$.

3. The number of $v_{i}-v_{j}(i \neq j)$ paths of length 2 in $\mathrm{G}$ is $a_{i j}^{(2)}$.

\section{Number of paths of length 3}

In this section, we give formulae to count the number of paths of length 3 in a simple graph G.

Theorem 3.1 Let $G$ be a simple graph with $n$ vertices and the adjacency matrix $A=\left[a_{i j}\right]$. The number of paths of length 3 in $G$ is $\sum_{i \neq j}\left(a_{i j}^{(3)}-\left(2 d_{j}-1\right) a_{i j}\right)$.

Proof: By Proposition 1.2, the number of paths of length 3 in $\mathrm{G}$ is equal to $\sum_{i \neq j} a_{i j}^{(3)}-\mathrm{x}$, where $\mathrm{x}$ is the number of non-closed walks of length 3 , that are not paths. To find $\mathrm{x}$, we have 2 cases as considered below; the cases are based on the configurations-(subgraphs) that generate all non-closed walks of length 3 , that are not paths. In each case, $\mathrm{N}$ denotes the number of non-closed walks of length 3, that are not paths in the corresponding subgraph, $\mathrm{M}$ denotes the number of subgraphs of $\mathrm{G}$ of the same configurations, $\mathrm{F}$ denotes the total number of non-closed walks of length 3, that are not paths in all possible subgraphs of $\mathrm{G}$ of the same configurations. It is clear that $\mathrm{F}$ is equal to $\mathrm{N} \times \mathrm{M}$. To find $\mathrm{N}$ in each case, we have to include in any walk, all the edges and the vertices of the corresponding subgraphs at least once.

Case 1: For the configuration of Fig $1, \mathrm{~N}=2, \mathrm{M}=\frac{1}{2} \sum_{i \neq j} a_{i j}$ and $\mathrm{F}=\sum_{i \neq j} a_{i j}$. 
Fig 1

Case 2: For the configuration of Fig $2, \mathrm{~N}=4, \mathrm{M}=\frac{1}{2} \sum_{i \neq j} a_{i j}\left(d_{j}-1\right)$ and $\mathrm{F}=2 \sum_{i \neq j} a_{i j}\left(d_{j}-1\right)$.

Fig 2

Consequently, $\mathrm{x}=\sum_{i \neq j} a_{i j}+2 \sum_{i \neq j} a_{i j}\left(d_{j}-1\right)$ and by simplifying, we get the desired result.

Example 3.2 In the graph of Fig 3, $\sum_{i \neq j}\left(a_{i j}^{(3)}-\left(2 d_{j}-1\right) a_{i j}\right)=24$. So, by Theorem 3.1, the number of paths of length 3 in $K_{4}$ is 24 .

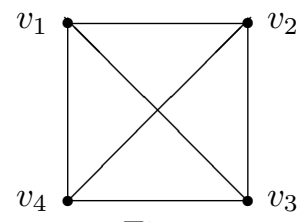

Fig 3

Theorem 3.3 Let $G$ be a simple graph with $n$ vertices and the adjacency matrix $A=\left[a_{i j}\right]$. The number of paths of length 3 in $G$, each of which starts from an specific vertex $v_{i}$ is $\sum_{j=1, i \neq j}^{n}\left(a_{i j}^{(3)}-\left(d_{i}+d_{j}-1\right) a_{i j}\right)$.

Proof: By Proposition 1.3, the number of paths of length 3 in $\mathrm{G}$, each of which starts from an specific vertex $v_{i}$ is $\sum_{j=1, i \neq j}^{n} a_{i j}^{(3)}-\mathrm{x}$, where $\mathrm{x}$ is the number of non-closed walks of length 3 , that are starting from $v_{i}$ and are not paths. To find $\mathrm{x}$, we have 3 cases as considered below; the cases are based on the configurations-(subgraphs) that generate all non-closed walks of length 3 , each of which starts from the specific vertex $v_{i}$, that are not paths. In each case, $\mathrm{N}$ denotes the number of non-closed walks of length 3 , which start from the vertex $v_{i}$ and are not paths in the corresponding subgraph, $\mathrm{M}$ denotes the number of subgraphs of $\mathrm{G}$ of the same configurations, $\mathrm{F}$ denotes the total number of non-closed walks of length 3 , which start from the vertex $v_{i}$ and are not paths in all possible subgraphs of $\mathrm{G}$ of the same configurations. It is clear that $\mathrm{F}$ is equal to $\mathrm{N} \times \mathrm{M}$. To find $\mathrm{N}$ in each case, we have to include in any walk, all the edges and the vertices of the corresponding subgraphs at least once.

Case 1: For the configuration of Fig $4, \mathrm{~N}=1, \mathrm{M}=\sum_{j=1, i \neq j}^{n} a_{i j}$ and $\mathrm{F}=\sum_{j=1, i \neq j}^{n} a_{i j}$.

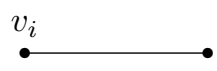

Fig 4

Case 2: For the configuration of Fig $5, \mathrm{~N}=1, \mathrm{M}=\sum_{j=1, i \neq j}^{n} a_{i j}\left(d_{j}-1\right)$ and $\mathrm{F}=\sum_{j=1, i \neq j}^{n} a_{i j}\left(d_{j}-1\right)$.

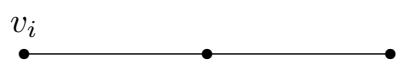

Fig 5

Case 3: For the configuration of Fig $6, \mathrm{~N}=2, \mathrm{M}=\frac{1}{2} \sum_{j=1, i \neq j}^{n} a_{i j}\left(d_{i}-1\right)$ and $\mathrm{F}=\sum_{j=1, i \neq j}^{n} a_{i j}\left(d_{i}-1\right)$.

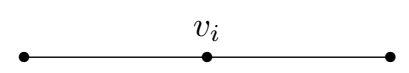

Fig 6 
Consequently, $\mathrm{x}=\sum_{j=1, i \neq j}^{n} a_{i j}+\sum_{j=1, i \neq j}^{n} a_{i j}\left(d_{j}-1\right)+\sum_{j=1, i \neq j}^{n} a_{i j}\left(d_{i}-1\right)$ and by simplifying, we get the desired result.

Example 3.4 In the graph of Fig 3, $\sum_{j=2}^{4}\left(a_{1 j}^{(3)}-\left(d_{1}+d_{j}-1\right) a_{1 j}\right)=6$. So, by Theorem 3.3, the number of paths of length 3, each of which starts from the vertex $v_{1}$, is 6 .

Theorem 3.5 Let $G$ be a simple graph with $n$ vertices and the adjacency matrix $A=\left[a_{i j}\right]$. The number of $v_{i}-v_{j}$ $(i \neq j)$ paths of length 3 in $G$ is $a_{i j}^{(3)}-\left(d_{i}+d_{j}-1\right) a_{i j}$.

Proof: By Proposition 1.4, the number of $v_{i}-v_{j}(i \neq j)$ paths of length 3 in $\mathrm{G}$ is equal to $a_{i j}^{(3)}-\mathrm{x}$, where $\mathrm{x}$ is the number of $v_{i}-v_{j}(i \neq j)$ walks of length 3 , that are not paths. To find $\mathrm{x}$, we have 3 cases as considered below; the cases are based on the configurations-(subgraphs) that generate $v_{i}-v_{j}(i \neq j)$ walks of length 3 that are not paths. In each case, $\mathrm{N}$ denotes the number of $v_{i}-v_{j}(i \neq j)$ walks of length 3 that are not paths in the corresponding subgraph, $\mathrm{M}$ denotes the number of subgraphs of $\mathrm{G}$ of the same configurations, $\mathrm{F}$ denotes the total number of $v_{i}-v_{j}$ $(i \neq j)$ walks of length 3 that are not paths in all possible subgraphs of $\mathrm{G}$ of the same configurations. It is clear that $\mathrm{F}$ is equal to $\mathrm{N} \times \mathrm{M}$. To find $\mathrm{N}$ in each case, we have to include in any walk, all the edges and the vertices of the corresponding subgraphs at least once.

Case 1: For the configuration of Fig $7, \mathrm{~N}=1, \mathrm{M}=a_{i j}$ and $\mathrm{F}=a_{i j}$.

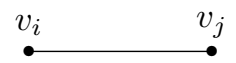

Fig 7

Case 2: For the configuration of $\mathrm{Fig} 8, \mathrm{~N}=1, \mathrm{M}=a_{i j}\left(d_{j}-1\right)$ and $\mathrm{F}=a_{i j}\left(d_{j}-1\right)$.

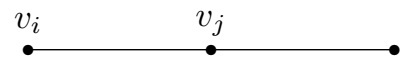

Fig 8

Case 3: For the configuration of Fig $9, \mathrm{~N}=1, \mathrm{M}=a_{i j}\left(d_{i}-1\right)$ and $\mathrm{F}=a_{i j}\left(d_{i}-1\right)$.

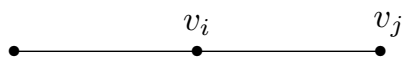

Fig 9

Consequently, $\mathrm{x}=a_{i j}+a_{i j}\left(d_{j}-1\right)+a_{i j}\left(d_{i}-1\right)$ and by simplifying, we get the desired result.

Example 3.6 In the graph of Fig 3, we have $a_{12}^{(3)}-\left(d_{1}+d_{2}-1\right) a_{12}=2$. So, by Theorem 3.5, the number of $v_{1}-v_{2}$ paths of length 3 is 2. Indeed, $v_{1} v_{4} v_{3} v_{2}$ and $v_{1} v_{3} v_{4} v_{2}$ are the two paths.

Now, we obtain other formulae for the number of paths of length 3.

Theorem 3.7 Let $G$ be a simple graph with $n$ vertices and the adjacency matrix $A=\left[a_{i j}\right]$. The number of paths of length 3 in $G$ is $\sum_{i \neq j} a_{i j}^{(2)}\left(d_{j}-a_{i j}-1\right)$.

Proof: Any path of length 3 in G, is obtainable from a path of length 2 by adding an edge to one of it's end vertices, provided by addition of this edge no triangle is formed. So, if we use $\sum_{i \neq j} a_{i j}^{(2)}\left(d_{j}-1\right)$ for the number of paths of length 3 in a graph $\mathrm{G}$, it will also contain all the subgraphs of $\mathrm{G}$, that are in the same configurations as the graph of Fig 10 for 6 times and the total number of subgraphs of $\mathrm{G}$ of the same configurations as the graph of Fig 10 is $\frac{1}{6} \sum_{i \neq j} a_{i j}^{(2)} a_{i j}$ (see Theorem 1.1). So, the number of paths of length 3 in a graph G is $\sum_{i \neq j} a_{i j}^{(2)}\left(d_{j}-1\right)-6 \times \frac{1}{6}$ $\sum_{i \neq j} a_{i j}^{(2)} a_{i j}$ and by simplifying, we get the desired result. 


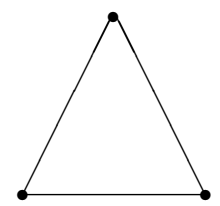

Fig 10

Example 3.8 In the graph of Fig 3, $\sum_{i \neq j} a_{i j}^{(2)}\left(d_{j}-a_{i j}-1\right)=24$. So, by Theorem 3.7, the number of paths of length 3 in $K_{4}$ is 24 .

Theorem 3.9 Let $G$ be a simple graph with $n$ vertices and the adjacency matrix $A=\left[a_{i j}\right]$. The number of paths of length 3 in $G$, each of which starts from an specific vertex $v_{i}$ is $\sum_{j=1, i \neq j}^{n} a_{i j}^{(2)}\left(d_{j}-a_{i j}-1\right)$.

Proof: Any path of length 3 that starts from an specific vertex $v_{i}$ in $\mathrm{G}$, is obtainable from a path of length 2 that starts from the vertex $v_{i}$ by adding an edge to it's end vertex, provided by addition of this edge no triangle is formed. So, if we use $\sum_{j=1, i \neq j}^{n} a_{i j}^{(2)}\left(d_{j}-1\right)$ for the number of paths of length 3 that are starting from the vertex $v_{i}$ in a graph $\mathrm{G}$, it will also contain all the subgraphs of $\mathrm{G}$, that are in the same configurations as the graph of Fig 11 for 2 times and the total number of subgraphs of $\mathrm{G}$ of the same configurations as the graph of Fig 11 is $\frac{1}{2} \sum_{j=1, i \neq j} a_{i j}^{(2)} a_{i j}$ (see Theorem 1.1). So, the number of paths of length 3 in a graph G which start from the vertex $v_{i}$ is $\sum_{j=1, i \neq j} a_{i j}^{(2)}\left(d_{j}-1\right)-2 \times \frac{1}{2} \sum_{j=1, i \neq j} a_{i j}^{(2)} a_{i j}$ and by simplifying, we get the desired result.

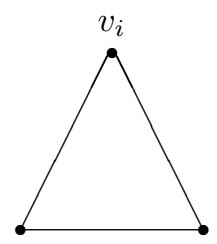

Fig 11

Example 3.10 In the graph of Fig 3, $\sum_{j=2}^{4} a_{1 j}^{(2)}\left(d_{j}-a_{1 j}-1\right)=6$. So, by Theorem 3.9, the number of paths of length 3, each of which starts from the vertex $v_{1}$, is 6 .

Theorem 3.11 Let $G$ be a simple graph with $n$ vertices and the adjacency matrix $A=\left[a_{i j}\right]$. The number of $v_{i}-v_{j}$ $(i \neq j)$ paths of length 3 in $G$ is $\sum_{k=1, k \neq i, j}^{n}\left(a_{i k}^{(2)}-a_{i j}\right) a_{j k}$.

Proof: Any $v_{i}-v_{j}(i \neq j)$ path of length 3 in $\mathrm{G}$, is obtainable from a $v_{i}-v_{k}(i \neq k$ and $k=1,2, \ldots, n)$ path of length 2 and a $v_{k}-v_{j}(k \neq j)$ path of length one. So, if we use $\sum_{k=1, k \neq i, j}^{n} a_{i k}^{(2)} a_{j k}$ for the number of $v_{i}-v_{j}(i \neq j)$ paths of length 3 in a graph $\mathrm{G}$, it will also contain all the subgraphs of $\mathrm{G}$ that are in the same configurations as the graph of Fig 12 for 1 time and Fig 12 is not the configuration of a path of length 3, that we don't want. The total number of subgraphs of $\mathrm{G}$ of the same configurations as the graph of Fig 12 is $\sum_{k=1, k \neq i, j}^{n} a_{i j} a_{j k}$. So, the number of $v_{i}-v_{j}$ paths of length 3 in a graph $\mathrm{G}$ is $\sum_{k=1, k \neq i, j}^{n} a_{i k}^{(2)} a_{j k}-\sum_{k=1, k \neq i, j}^{n} a_{i j} a_{j k}$ and by simplifying, we get the desired result.

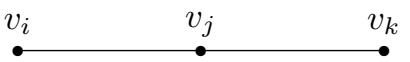

Fig 12 
Example 3.12 In the graph of Fig 3, $\sum_{k=3}^{4}\left(a_{1 k}^{(2)}-a_{12}\right) a_{2 k}=2$. So, by Theorem 3.11, the number of $v_{1}-v_{2}$ paths of length 3 is 2. Indeed, $v_{1} v_{4} v_{3} v_{2}$ and $v_{1} v_{3} v_{4} v_{2}$ are the two paths.

\section{Number of paths of length 4}

In this section, we give formulae to count the number of paths of length 4 in a simple graph $\mathrm{G}$.

Theorem 4.1 Let $G$ be a simple graph with $n$ vertices and the adjacency matrix $A=\left[a_{i j}\right]$. The number of paths of length 4 in $G$ is $\sum_{i \neq j}\left[a_{i j}^{(4)}-2 a_{i j}^{(2)}\left(d_{j}-a_{i j}\right)\right]-\sum_{i=1}^{n}\left[\left(2 d_{i}-1\right) a_{i i}^{(3)}+6\left(\begin{array}{c}d_{i} \\ 3\end{array}\right)\right]$.

Proof: By Proposition 1.2, the number of paths of length 4 in a graph $\mathrm{G}$ is equal to $\sum_{i \neq j} a_{i j}^{(4)}-\mathrm{x}$, where $\mathrm{x}$ is the number of non-closed walks of length 4 , that are not paths. To find $\mathrm{x}$, we have 5 cases as considered below; the cases are based on the configurations-(subgraphs) that generate all non-closed walks of length 4 , that are not paths. In each case, $\mathrm{N}$ denotes the number of non-closed walks of length 4, that are not paths in the corresponding subgraph, $\mathrm{M}$ denotes the number of subgraphs of $\mathrm{G}$ of the same configurations and $\mathrm{F}$ denotes the total number of non-closed walks of length 4 , that are not paths in all possible subgraphs of $\mathrm{G}$ of the same configurations. It is clear that $\mathrm{F}$ is equal to $\mathrm{N} \times \mathrm{M}$. To find $\mathrm{N}$ in each case, we have to include in any walk, all the edges and the vertices of the corresponding subgraphs at least once.

Case 1: For the configuration of Fig 13, N=4, M= $\frac{1}{2} \sum_{i \neq j} a_{i j}^{(2)}$ and $\mathrm{F}=2 \sum_{i \neq j} a_{i j}^{(2)}$.

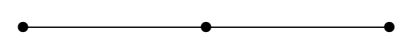

Fig 13

Case 2: For the configuration of Fig $14, \mathrm{~N}=4, \mathrm{M}=\frac{1}{2} \sum_{i \neq j} a_{i j}^{(2)}\left(d_{j}-a_{i j}-1\right)$ and $\mathrm{F}=2 \sum_{i \neq j} a_{i j}^{(2)}\left(d_{j}-a_{i j}-1\right)$ (see Theorem 3.7).

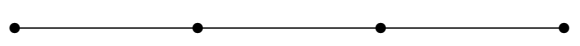

Fig 14

Case 3: For the configuration of Fig 15, $\mathrm{N}=6, \mathrm{M}=\sum_{i=1}^{n}\left(\begin{array}{c}d_{i} \\ 3\end{array}\right)$ and $\mathrm{F}=6 \sum_{i=1}^{n}\left(\begin{array}{c}d_{i} \\ 3\end{array}\right)$.

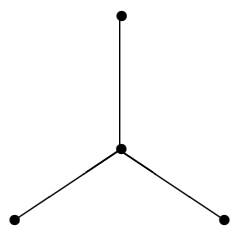

Fig 15

Case 4: For the configuration of Fig 16, $\mathrm{N}=4, \mathrm{M}=\frac{1}{2} \sum_{i=1}^{n} a_{i i}^{(3)}\left(d_{i}-2\right)$ and $\mathrm{F}=2 \sum_{i=1}^{n} a_{i i}^{(3)}\left(d_{i}-2\right)$ (see Theorem 1.1).

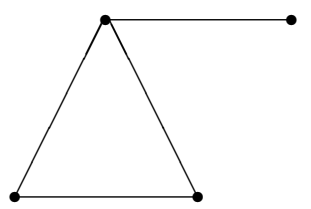

Fig 16 
Case 5: For the configuration of Fig $17, \mathrm{~N}=18, \mathrm{M}=\frac{1}{6} \sum_{i=1}^{n} a_{i i}^{(3)}$ and $\mathrm{F}=3 \sum_{i=1}^{n} a_{i i}^{(3)}$ (see Theorem 1.1).

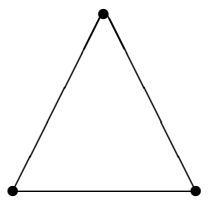

Fig 17

Now we add the values of $\mathrm{F}$ arising from the above cases and determine $\mathrm{x}$. By putting the value of $\mathrm{x}$ in $\sum_{i \neq j} a_{i j}^{(4)}-\mathrm{x}$ and simplifying, we get the desired result.

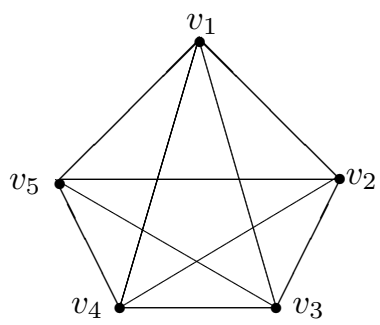

Fig 18

Example 4.2 In the graph of Fig 18, $\sum_{i \neq j}\left[a_{i j}^{(4)}-2 a_{i j}^{(2)}\left(d_{j}-a_{i j}\right)\right]=660$ and $\sum_{i=1}^{5}\left[\left(2 d_{i}-1\right) a_{i i}^{(3)}+6\left(\begin{array}{c}d_{i} \\ 3\end{array}\right)\right]=540$. So, by Theorem 4.1, the number of paths of length 4 is 120.

Theorem 4.3 Let $G$ be a simple graph with $n$ vertices and the adjacency matrix $A=\left[a_{i j}\right]$. The number of paths of length 4 in $G$, each of which starts from an specific vertex $v_{i}$ is $\sum_{j=1, i \neq j}^{n}\left[a_{i j}^{(4)}-\left(d_{i}+d_{j}-3 a_{i j}\right) a_{i j}^{(2)}-\left(a_{i i}^{(3)}+a_{j j}^{(3)}+\right.\right.$ $\left.\left.2\left(\begin{array}{c}d_{j}-1 \\ 2\end{array}\right)\right) a_{i j}\right]$

Proof: By Proposition 1.3, the number of paths of length 4 in a graph G, each of which starts from an specific vertex $v_{i}$ is $\sum_{j=1, i \neq j}^{n} a_{i j}^{(4)}-\mathrm{x}$, where $\mathrm{x}$ is the number of non-closed walks of length 4 , that begin from $v_{i}$ and are not paths. To find $\mathrm{x}$, we have 7 cases as considered below; the cases are based on the configurations-(subgraphs) that generate all non-closed walks of length 4 , each of which starts from the specific vertex $v_{i}$, that are not paths. In each case, $\mathrm{N}$ denotes the number of non-closed walks of length 4 , which start from the vertex $v_{i}$ and are not paths in the corresponding subgraph, $\mathrm{M}$ denotes the number of subgraphs of $\mathrm{G}$ of the same configurations, $\mathrm{F}$ denotes the total number of non-closed walks of length 4 , which start from the vertex $v_{i}$ and are not paths in all possible subgraphs of $\mathrm{G}$ of the same configurations. However, in the cases with more than one figure (cases 3, 6, 7), N, M and F are based on the first graph of the respective figures and $\mathrm{P}$ denotes the number of subgraphs of G which don't have the same configurations as the first graph but are counted in $\mathrm{M}$. It is clear that $\mathrm{F}$ is equal to $\mathrm{N} \times(\mathrm{M}-\mathrm{P})$. To find $\mathrm{N}$ in each case, we have to include in any walk, all the edges and the vertices of the corresponding subgraphs at least once.

Case 1: For the configuration of Fig 19, $\mathrm{N}=2, \mathrm{M}=\sum_{j=1, i \neq j}^{n} a_{i j}^{(2)}$ and $\mathrm{F}=2 \sum_{j=1, i \neq j}^{n} a_{i j}^{(2)}$.

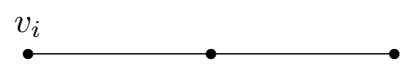

Fig 19

Case 2: For the configuration of Fig 20, $\mathrm{N}=1, \mathrm{M}=\sum_{j=1, i \neq j}^{n} a_{i j}^{(2)}\left(d_{j}-a_{i j}-1\right)$ and $\mathrm{F}=\sum_{j=1, i \neq j}^{n} a_{i j}^{(2)}\left(d_{j}-a_{i j}-1\right)$ (see Theorem 3.9). 


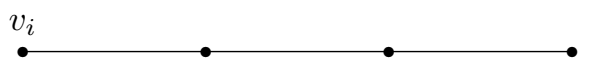

Fig 20

Case 3: For the configuration of Fig $21(\mathrm{a}), \mathrm{N}=1, \mathrm{M}=\sum_{j=1, i \neq j}^{n} a_{i j}^{(2)}\left(d_{i}-1\right)$. Let $\mathrm{P}$ denotes the number of all subgraphs of $\mathrm{G}$ that have the same configurations as the graph of Fig $21(\mathrm{~b})$ and are counted in M. Thus $\mathrm{P}=2 \times \frac{1}{2} \sum_{j=1, i \neq j}^{n} a_{i j}^{(2)} a_{i j}$, where $\frac{1}{2} \sum_{j=1, i \neq j}^{n} a_{i j}^{(2)} a_{i j}$ is the number of subgraphs of $\mathrm{G}$ that have the same configurations as the graph of Fig 21(b) and 2 is the number of times that this subgraph is counted in M. Consequently, $\mathrm{F}=\sum_{j=1, i \neq j}^{n} a_{i j}^{(2)}\left(d_{i}-a_{i j}-1\right)$.

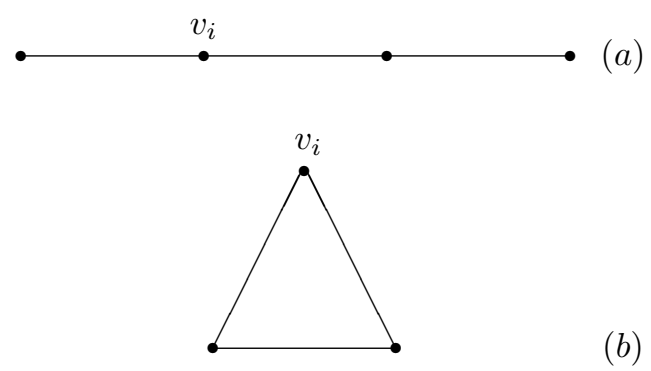

Fig 21

Case 4: For the configuration of Fig $22, \mathrm{~N}=2, \mathrm{M}=\sum_{j=1, i \neq j}^{n}\left(\begin{array}{c}d_{j}-1 \\ 2\end{array}\right) a_{i j}$ and $\mathrm{F}=2 \sum_{j=1, i \neq j}^{n}\left(\begin{array}{c}d_{j}-1 \\ 2\end{array}\right) a_{i j}$.

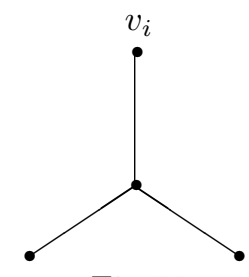

Fig 22

Case 5: For the configuration of Fig 23, $\mathrm{N}=6, \mathrm{M}=\frac{1}{2} \sum_{j=1, i \neq j}^{n} a_{i j}^{(2)} a_{i j}$ and $\mathrm{F}=3 \sum_{j=1, i \neq j}^{n} a_{i j}^{(2)} a_{i j}$.

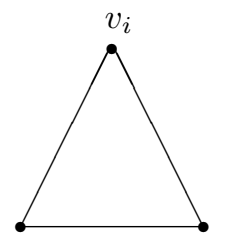

Fig 23

Case 6: For the configuration of Fig $24(a), \mathrm{N}=2, \mathrm{M}=\frac{1}{2} \sum_{j=1, i \neq j}^{n} a_{i i}^{(3)} a_{i j}$. Let $\mathrm{P}$ denotes the number of all subgraphs of $\mathrm{G}$ that have the same configurations as the graph of Fig $24(\mathrm{~b})$ and are counted in M. Thus $\mathrm{P}=2 \times \frac{1}{2} \sum_{j=1, i \neq j}^{n} a_{i j}^{(2)} a_{i j}$, where $\frac{1}{2} \sum_{j=1, i \neq j}^{n} a_{i j}^{(2)} a_{i j}$ is the number of subgraphs of $\mathrm{G}$ that have the same configurations as the graph of Fig 24(b) 
and 2 is the number of times that this subgraph is counted in M. Consequently, $\mathrm{F}=\sum_{j=1, i \neq j}^{n} a_{i i}^{(3)} a_{i j}-2 \sum_{j=1, i \neq j}^{n} a_{i j}^{(2)} a_{i j}$.

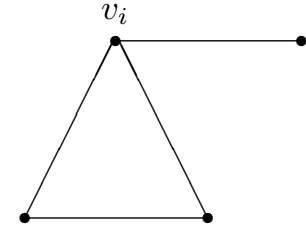

(a)

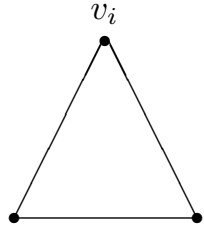

(b)

Fig 24

Case 7: For the configuration of Fig $25(a), \mathrm{N}=2, \mathrm{M}=\frac{1}{2} \sum_{j=1, i \neq j}^{n} a_{j j}^{(3)} a_{i j}$. Let $\mathrm{P}$ denotes the number of all subgraphs of G that have the same configurations as the graph of Fig $25(\mathrm{~b})$ and are counted in M. Thus $\mathrm{P}=2 \times \frac{1}{2} \sum_{j=1, i \neq j}^{n} a_{i j}^{(2)} a_{i j}$, where $\frac{1}{2} \sum_{j=1, i \neq j}^{n} a_{i j}^{(2)} a_{i j}$ is the number of subgraphs of G that have the same configurations as the graph of Fig 25(b) and 2 is the number of times that this subgraph is counted in M. Consequently, $\mathrm{F}=\sum_{j=1, i \neq j}^{n} a_{j j}^{(3)} a_{i j}-2 \sum_{j=1, i \neq j}^{n} a_{i j}^{(2)} a_{i j}$.

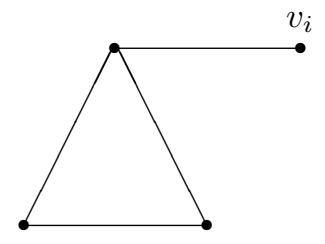

(a)

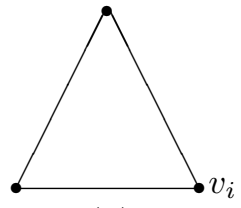

(b)

Fig 25

Now we add the values of $\mathrm{F}$ arising from the above cases and determine $\mathrm{x}$. By putting the value of $\mathrm{x}$ in $\sum_{j=1, i \neq j}^{n} a_{i j}^{(4)}-\mathrm{x}$ and simplifying, we get the desired result.

Example 4.4 In the graph of Fig 18, $\sum_{j=2}^{5}\left[a_{1 j}^{(4)}-\left(d_{1}+d_{j}-3 a_{1 j}\right) a_{1 j}^{(2)}-\left(a_{11}^{(3)}+a_{j j}^{(3)}+2\left(\begin{array}{c}d_{j}-1 \\ 2\end{array}\right)\right) a_{1 j}\right]=24$. So, by Theorem 4.3, the number of paths of length 4 starting from the vertex $v_{1}$ is 24.

Theorem 4.5 Let $G$ be a simple graph with $n$ vertices and the adjacency matrix $A=\left[a_{i j}\right]$. The number of $v_{i}-v_{j}$ paths of length 4 in $G$ is $a_{i j}^{(4)}-\left(d_{i}+d_{j}-3 a_{i j}\right) a_{i j}^{(2)}-\left(a_{i i}^{(3)}+a_{j j}^{(3)}\right) a_{i j}-\sum_{k=1, k \neq i, j}^{n} a_{i k} a_{k j}\left(d_{k}-2\right)$.

Proof: By Proposition 1.6, the number of $v_{i}-v_{j}(i \neq j)$ paths of length 4 in a graph $\mathrm{G}$ is $a_{i j}^{(4)}-\mathrm{x}$, where $\mathrm{x}$ is the number of $v_{i}-v_{j}(i \neq j)$ walks of length 4 , that are not paths. To find $\mathrm{x}$, we have 7 cases as considered below; the cases are based on the configurations-(subgraphs) that generate $v_{i}-v_{j}(i \neq j)$ walks of length 4 , that are not paths. In each case, $\mathrm{N}$ denotes the number of $v_{i}-v_{j}(i \neq j)$ walks of length 4 that are not paths in the corresponding subgraph, $\mathrm{M}$ denotes the number of subgraphs of $\mathrm{G}$ of the same configurations, F denotes the total number of $v_{i}-v_{j}$ $(i \neq j)$ walks of length 4 that are not paths in all possible subgraphs of $\mathrm{G}$ of the same configurations. However, in the cases with more than one figure (cases 2, 3, 6, 7), N, M and F are based on the first graph of the respective figures and $\mathrm{P}$ denotes the number of subgraphs of $\mathrm{G}$ which don't have the same configurations as the first graph but are counted in $\mathrm{M}$. It is clear that $\mathrm{F}$ is equal to $\mathrm{N} \times(\mathrm{M}-\mathrm{P})$. To find $\mathrm{N}$ in each case, we have to include in any walk, all the edges and the vertices of the corresponding subgraphs at least once.

Case 1: For the configuration of Fig $26, \mathrm{~N}=2, \mathrm{M}=a_{i j}^{(2)}$ and $\mathrm{F}=2 a_{i j}^{(2)}$.

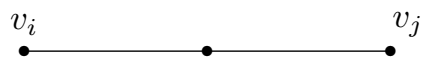

Fig 26 
Case 2: For the configuration of Fig $27(\mathrm{a}), \mathrm{N}=1, \mathrm{M}=a_{i j}^{(2)}\left(d_{j}-1\right)$. Let $\mathrm{P}$ denotes the number of all subgraphs of $\mathrm{G}$ that have the same configurations as the graph of Fig $27(\mathrm{~b})$ and are counted in M. Thus $\mathrm{P}=1 \times a_{i j}^{(2)} a_{i j}$, where $a_{i j}^{(2)} a_{i j}$ is the number of subgraphs of $\mathrm{G}$ that have the same configurations as the graph of Fig 27(b) and 1 is the number of times that this subgraph is counted in M. Consequently, $\mathrm{F}=a_{i j}^{(2)}\left(d_{j}-a_{i j}-1\right)$.
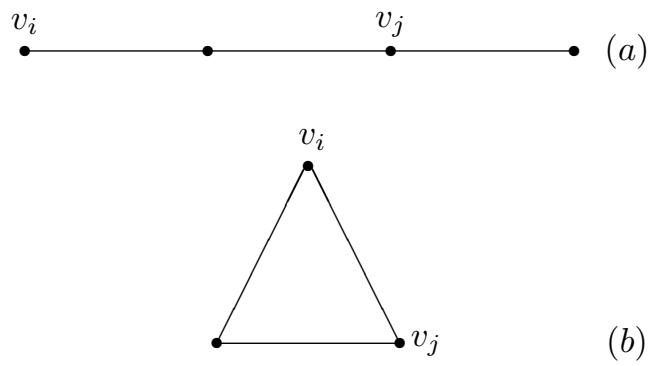

Fig 27

Case 3: For the configuration of Fig $28(\mathrm{a}), \mathrm{N}=1, \mathrm{M}=a_{i j}^{(2)}\left(d_{i}-1\right)$. Let $\mathrm{P}$ denotes the number of all subgraphs of $\mathrm{G}$ that have the same configurations as the graph of Fig $28(\mathrm{~b})$ and are counted in M. Thus $\mathrm{P}=1 \times a_{i j}^{(2)} a_{i j}$, where $a_{i j}^{(2)} a_{i j}$ is the number of subgraphs of $\mathrm{G}$ that have the same configurations as the graph of Fig 28(b) and 1 is the number of times that this subgraph is counted in M. Consequently, $\mathrm{F}=a_{i j}^{(2)}\left(d_{i}-a_{i j}-1\right)$.
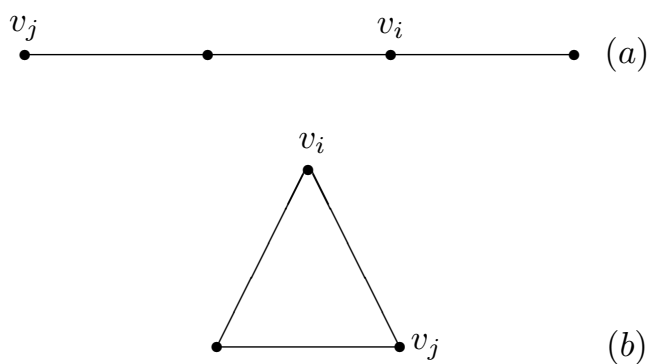

a)

Fig 28

Case 4: For the configuration of Fig 29, $\mathrm{N}=1, \mathrm{M}=\sum_{k=1, k \neq i, j}^{n} a_{i k} a_{k j}\left(d_{k}-2\right)$ and $\mathrm{F}=\sum_{k=1, k \neq i, j}^{n} a_{i k} a_{k j}\left(d_{k}-2\right)$.

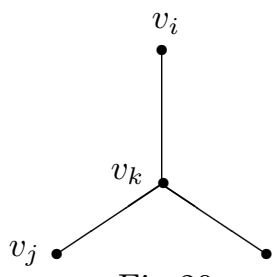

Fig 29

Case 5: For the configuration of Fig $30, \mathrm{~N}=3, \mathrm{M}=a_{i j}^{(2)} a_{i j}$ and $\mathrm{F}=3 a_{i j}^{(2)} a_{i j}$.

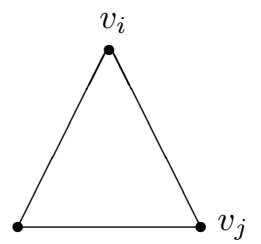

Fig 30 
Case 6: For the configuration of Fig $31(a), \mathrm{N}=2, \mathrm{M}=\frac{1}{2} a_{i i}^{(3)} a_{i j}$. Let $\mathrm{P}$ denotes the number of all subgraphs of $\mathrm{G}$ that have the same configurations as the graph of Fig $31(\mathrm{~b})$ and are counted in $\mathrm{M}$. Thus $\mathrm{P}=1 \times a_{i j}^{(2)} a_{i j}$, where $a_{i j}^{(2)} a_{i j}$ is the number of subgraphs of $\mathrm{G}$ that have the same configurations as the graph of Fig 31(b) and 1 is the number of times that this subgraph is counted in M. Consequently, $\mathrm{F}=a_{i i}^{(3)} a_{i j}-2 a_{i j}^{(2)} a_{i j}$.

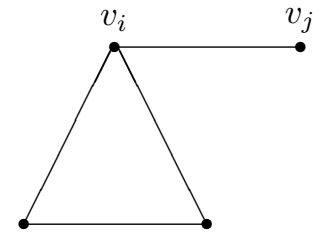

(a)

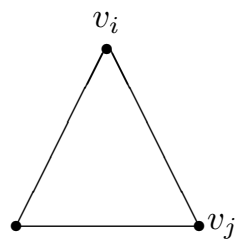

(b)

Fig 31

Case 7: For the configuration of Fig $32(a), \mathrm{N}=2, \mathrm{M}=\frac{1}{2} a_{j j}^{(3)} a_{i j}$. Let $\mathrm{P}$ denotes the number of all subgraphs of $\mathrm{G}$ that have the same configurations as the graph of Fig $32(\mathrm{~b})$ and are counted in M. Thus $\mathrm{P}=1 \times a_{i j}^{(2)} a_{i j}$, where $a_{i j}^{(2)} a_{i j}$ is the number of subgraphs of $\mathrm{G}$ that have the same configurations as the graph of Fig 32(b) and 1 is the number of times that this subgraph is counted in M. Consequently, $\mathrm{F}=a_{j j}^{(3)} a_{i j}-2 a_{i j}^{(2)} a_{i j}$.

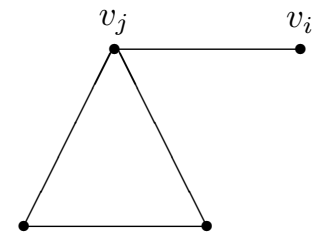

(a)

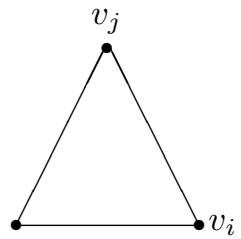

(b)

Fig 32

Now we add the values of $\mathrm{F}$ arising from the above cases and determine $\mathrm{x}$. By putting the value of $\mathrm{x}$ in $a_{i j}^{(4)}-\mathrm{x}$ and simplifying, we get the desired result.

Example 4.6: In the graph of Fig 18, $a_{12}^{(4)}=51,\left(d_{1}+d_{2}-3 a_{12}\right) a_{12}^{(2)}=15,\left(a_{11}^{(3)}+a_{22}^{(3)}\right) a_{12}=24$, $\sum_{k=3}^{5} a_{1 k} a_{k 2}\left(d_{k}-2\right)=6$. So, by Theorem 4.5 , the number of $v_{1}-v_{2}$ paths of length 4 is 6 .

\section{References}

[1] F. Harary and B. Manvel, On the number of cycles in a graph, Mat. Casopis Sloven. Akad. Vied 21 (1971),55-63.

[2] I.Tomescu, On the number of paths and cycles for almost all graphs and digraphs, Combinatorica, 6(1) (1986), 73-79.

[3] Eric T. Bax, Algorithms to count paths and cycles, Inf. Process. Lett, 52 (1994), 249-252.

[4] Noga Alon, Raphy Yuster and Uri Zwick, Color-coding: a new method for finding simple paths, cycles and other small subgraphs within large graphs, ACM Newyork, 1994, 326-335.

[5] Eric Bax and Joel Franklin, A finite-difference sieve to count paths and cycles by length, Inf. Process. Lett, 60(4) (1996), 171-176.

[6] Ben Roberts and Drik P. Kroese, Estimating the number of s-t paths in a graph, J. Graph Algorithms and Applications, 11(1) (2007), 195-214.

[7] Ryan Williams, Finding a path of length $\mathrm{k}$ in $O^{*}\left(2^{K}\right)$ time, Inf. Process. Lett, 109(6) (2009), 315-318.

[8] Andreas Bjorklund, Thore Husfeldt, Petteri Kaski and Mikko Koivisto, Counting paths and packing in halves, Lecture Notes in Computer Science, 5757 (2009), 578-586.

[9] Eric. T. Bax, Inclusion and exclusion algorithm for the Hamiltonian path problem, Inform. Process. Lett. 27(4) (1993), 203-207. 
[10] A. Bjorklund, T. Husfeldt, P. Kaski, M. Koivisto, The fast intersection transform with applications to counting paths, CoRR, abs/0809.2489 (2008).

[11] J. Chen, S. Lu, S. H. Sze, F. Zhang, Improved algorithms for path, matching and packing problems, 18th Annual ACMSIAM Symposium on Discrete Algorithms (SODA 2007), Philadelphia, PA, USA, PP. 298-307. Society for Industrial and Applied Mathematics (2007).

[12] Y. Gurevich, S. Shelah, Expected computation time for Hamiltonian path problem, SIAM J. Comput, 16(1987), 486-502.

[13] I. Koutis, Faster algebraic algorithm for path and packing problems, CALP (2008), LNCS 5125, 575-586, Springer.

[14] D. J. A. Welsh, Complexity: Konts, Colouring and counting, Cambridge University Press, 1993.

[15] Eric. T. Bax, Improvements and bounding for the inclusion and exclusion Hamiltonian algorithm, Caltech-CS-TR-94-11.

[16] B. Monien, How to find long paths efficiently, Annals of Discrete Mathematics, volume 25, (1985), $239-254$.

[17] D. Richards, Finding short cycles in a planar graph using separators, Journal of Algorithms, volume 7, (1974), 382-394.

[18] J.A. Bondy and M. Simonovit, Cycles of even length in graphs, Journal of Combinatorial Theory Series B, volume 16, (1974), 97-105.

[19] Lowell W. Beineke and Robin J. Wilson, Topics in Algebraic Graph Theory, Cambridge University Press, 2007.

[20] F. Harary, Graph Theory, Addison-Wesley, Reading, Mass, 1969.

[21] G. Baroti, On the number of certain hamilton circuits of a complete graph, Periodica Math. Hung, 3 (1973), 135-139. 